\title{
Symbol Estimation Algorithm for MIMO Underwater Acoustic Communication System Based on Multiplicative Noise Model
}

\author{
Ling Zhang, ${ }^{1}$ Ming Li, ${ }^{1}$ Gangsheng Li, ${ }^{2}$ and Rui Wang ${ }^{1}$ \\ ${ }^{1}$ College of Engineering, Ocean University of China, Qingdao 266100, China \\ ${ }^{2}$ Department of Education, Ocean University of China, Qingdao 266100, China \\ Correspondence should be addressed to Ming Li; limingneu@ouc.edu.cn
}

Received 25 September 2014; Accepted 22 January 2015

Academic Editor: Hsuan-Ling Kao

Copyright (c) 2015 Ling Zhang et al. This is an open access article distributed under the Creative Commons Attribution License, which permits unrestricted use, distribution, and reproduction in any medium, provided the original work is properly cited.

\begin{abstract}
The stochastic and time-varying underwater acoustic (UWA) channels are usually affected by serious multipath delays, energy loss and distortion factors, thus making the modeling and estimation of the UWA channel challenging problems in the research community. Based on the analysis of the UWA channel, the system with multiplicative noise (SMN) model is established to characterize the complicated factors such as random time-variation, nonlinearity, and energy attenuation. As to the multiple-input multiple-output (MIMO) UWA communication, the complicated SMN model is established for MIMO UWA channels; based on which, the transmitted symbols are estimated according to the optimal recursive filtering algorithm. The algorithm is derived based on the projection theorem, which is optimal in the sense of linear minimum variance, and can overcome the intersymbol interference and noise pollution efficiently. The optimal algorithm is computed recursively, which has the advantage of computationefficiency and can track the random variation of the fast time-varying channel gain dynamically. Simulation results have validated the effectiveness of the algorithm. The model and the algorithm can be extended flexibly to certain practical problems, such as the joint channel and symbol estimation in underwater acoustic communication systems.
\end{abstract}

\section{Introduction}

Underwater acoustic (UWA) communication usually works in very complex transmission environment with seriously noisy background and very limited bandwidth. In addition, the complex multipath propagation and time-frequency selective fading are prone to causing serious distortion of UWA communication signals, which set obstacles to the high-speed and reliable transmission of UWA communication. Hence, how to characterize the underwater channel and estimate the transmitted symbol have become the focus of current research [1-4].

In recent years, there is an increasing demand for high data rate underwater acoustic communication. Thus, some advanced and sophisticated technologies from land wireless communication are introduced into the field of underwater coherent communication, such as multiple input multiple output (MIMO) technology [5-8] and orthogonal frequency division multiplexing (OFDM) technology [9-11]. MIMO technology especially fully exploits space resources and transmits signals from various transmitting and receiving apparatus occupying the same frequency band, without additional receiver transmitting power and spectrum resource; therefore it can greatly increase channel capacity and reduce the bit error rate (BER) of system.

Underwater acoustic channel is an integral part of UWA communication system. Considering the various constraints and the time-space-frequency variability of the channel, UWA channel is much more complex than the radio channel. Usually, there exist reflection and refraction in the propagation of acoustic wave in seawater as marine environment is inhomogeneous, which results in a lot of acoustic signals superimposed in the receiving end and the so-called multipath effect leads to intersymbol interference (ISI) and signal amplitude fading. Currently, the adaptive equalization technology is commonly used to overcome this interference 
[12-15]. But the equalization algorithm cannot perform well under complex and random time-varying communication environment.

Recently, the research on the multiplicative noise in UWA communication has become a hot spot [16-19] because the multiplicative noise can describe many factors in practical systems such as nonlinearity, packet dropout, and frequency offset. Considering the various kinds of interference in UWA channel, it can be characterized by a system with multiplicative noise (SMN) model. In [20, 21], the UWA channel is seen as SMN with additive and multiplicative noises, and the underwater target is regarded as a special scatterer. Therefore, the problem of underwater target detection can be converted into the estimation of the scattering coefficient sequence constituted by the target and the scatterer nearby. Conventionally, the SMN model is applied in single input multiple output (SIMO) UWA system and seldom considers the MIMO system. This paper focuses on the modeling of MIMO UWA channel and derives the optimal estimation algorithm based on the new model, which can be applied for more complex UWA communication system.

In this paper, based on the characteristics of the UWA channel, the multiplicative noise is introduced to model the MIMO UWA communication system in order to track the fast time-varying UWA channel dynamically; that is, a novel state-space model with multiplicative noise is established to describe the channel variation and achieve effective estimations of the transmitted symbols. Based on the system model, the optimal recursive filtering algorithm is derived for complicated multichannel system with multiplicative noise in the form of general stochastic matrix, which can solve the optimal estimation problem of systems under more complicated environment. The filtering algorithm is optimal under the linear minimum variance criterion and is computed recursively, which is suitable for high speed UWA communication. In the modeling process, the transmitted symbols are included in the state vector. Therefore, according to this correspondence between the state vector in the system model and the transmitted signals from various transmitters, the symbol detection problem can be converted into the filtering estimation of the system state.

The paper is arranged as follows. The SMN model is established to characterize the MIMO UWA channel in Section 2. In Section 3, the symbol estimation algorithm is developed for MIMO UWA system. The performance of the established model and the present algorithm is analyzed in Section 4. Simulation results are shown to validate the algorithm in Section 5. Finally, conclusions are drawn in Section 6.

\section{MIMO UWA Channel Modeling Based on SMN}

For an MIMO UWA communication system employing $N$ transducer sources and $M$ hydrophone receivers, the discrete-time baseband equivalent signal received at the $i$ th receiver is expressed by

$$
z_{i}(k)=\sum_{j=1}^{N} \sum_{l=0}^{L-1} s_{j}(k-l) h_{j, i}(k, l)+v_{i}(k),
$$

where $s_{j}(k)$ is the transmitted symbol from the $j$ th transducer at time instant $k ; h_{j, i}(k, l)$ is the $l$ th fading channel coefficient between the $j$ th transducer and the $i$ th receiver at time $k$; $L$ is the maximum length of channels; and $v_{i}(k)$ is the zero-mean additive white Gaussian noise on the $i$ th receiver.

If the UWA channel has ideal transmission characteristics, the slowly varying and stable compositions will be the dominant channel characteristics [22]; thereby the channel parameters remain unchanged during a packet or even few packets of data; that is, $h_{j, i}(k, l) \approx h_{j, i}(l)$. Thus (1) can be rewritten as

$$
z_{i}(k)=\sum_{j=1}^{N} \sum_{l=0}^{L-1} s_{j}(k-l) h_{j, i}(l)+v_{i}(k) .
$$

Otherwise, for underwater remote communication systems, UWA channel usually has more complex transmission characteristics [16]. Signals from transmitters along different paths arrive at the receiving end at different times. Therefore, the received signal is the superposition of all these signals, causing waveform distortion. This effect of UWA channel includes not only marine environment noise and target radiation noise as additive interferences, but also a variety of distortions and information energy attenuation as multiplicative interferences due to the random time-varying characteristics of UWA channel. Broadly speaking, those factors that bring the received signal multiplicative interference can be characterized by the multiplicative noise. Therefore, in this paper, based on the random characteristics of the UWA channel, the multiplicative noise is applied to characterize the complicated and time-varying UWA channel, and the convolution model with multiplicative noise in the received signal can be expressed as

$$
\begin{aligned}
z_{i}(k)= & \sum_{j=1}^{N} \sum_{l=0}^{L-1} s_{j}(k-l) m_{i, l+1}(k) h_{j, i}(l)+v_{i}(k) \\
= & \sum_{j=1}^{N} s_{j}(k) m_{i, 1}(k) h_{j, i}(0)+s_{j}(k-1) m_{i, 2}(k) h_{j, i}(1) \\
& +\cdots+s_{j}(k-L+1) m_{i, L}(k) h_{j, i}(L-1)+v_{i}(k)
\end{aligned}
$$

where the multiplicative noise $\left\{m_{i j}(k), 1 \leq i \leq M, 1 \leq j \leq L\right\}$ is of Gaussian distribution with the mathematic expectation $\mathrm{E}\left\{m_{i j}(k)\right\}=\bar{m}_{i j}(k)$ and known covariance.

From (3), let

$$
\begin{gathered}
\mathbf{x}(k)=\left[x_{1}(k) \cdots x_{L}(k), x_{L+1}(k) \cdots x_{2 L}(k), \ldots,\right. \\
\left.x_{(N-1) L+1}(k) \cdots x_{N L}(k)\right]^{T},
\end{gathered}
$$




$$
\begin{gathered}
s_{1}(k)=x_{1}(k), \ldots, s_{1}(k-L+1)=x_{L}(k), \\
s_{2}(k)=x_{L+1}(k), \ldots, s_{2}(k-L+1)=x_{2 L}(k)
\end{gathered}
$$

$$
s_{N}(k)=x_{(N-1) L+1}(k), \ldots, s_{N}(k-L+1)=x_{N L}(k) .
$$
define

For $N \times M$ underwater acoustic communication system,

$$
\begin{aligned}
& \mathbf{z}(k)=\left[\begin{array}{llll}
z_{1}(k) & z_{2}(k) & \cdots & z_{M}(k)
\end{array}\right]^{T} \\
& \mathbf{v}(k)=\left[\begin{array}{llll}
v_{1}(k) & v_{2}(k) & \cdots & v_{M}(k)
\end{array}\right]^{T}
\end{aligned}
$$

$$
\begin{aligned}
& \mathbf{A}=\left[\begin{array}{cccc}
\mathbf{A}_{1} & \mathbf{0} & \cdots & \mathbf{0} \\
\mathbf{0} & \mathbf{A}_{2} & \ddots & \vdots \\
\vdots & \ddots & \ddots & \mathbf{0} \\
\mathbf{0} & \cdots & \mathbf{0} & \mathbf{A}_{N}
\end{array}\right], \quad \mathbf{A}_{j}=\left[\begin{array}{cccc}
0 & 0 & \cdots & 0 \\
1 & 0 & \cdots & 0 \\
\vdots & \ddots & \ddots & \vdots \\
0 & \cdots & 1 & 0
\end{array}\right]_{(L \times L)}
\end{aligned}
$$

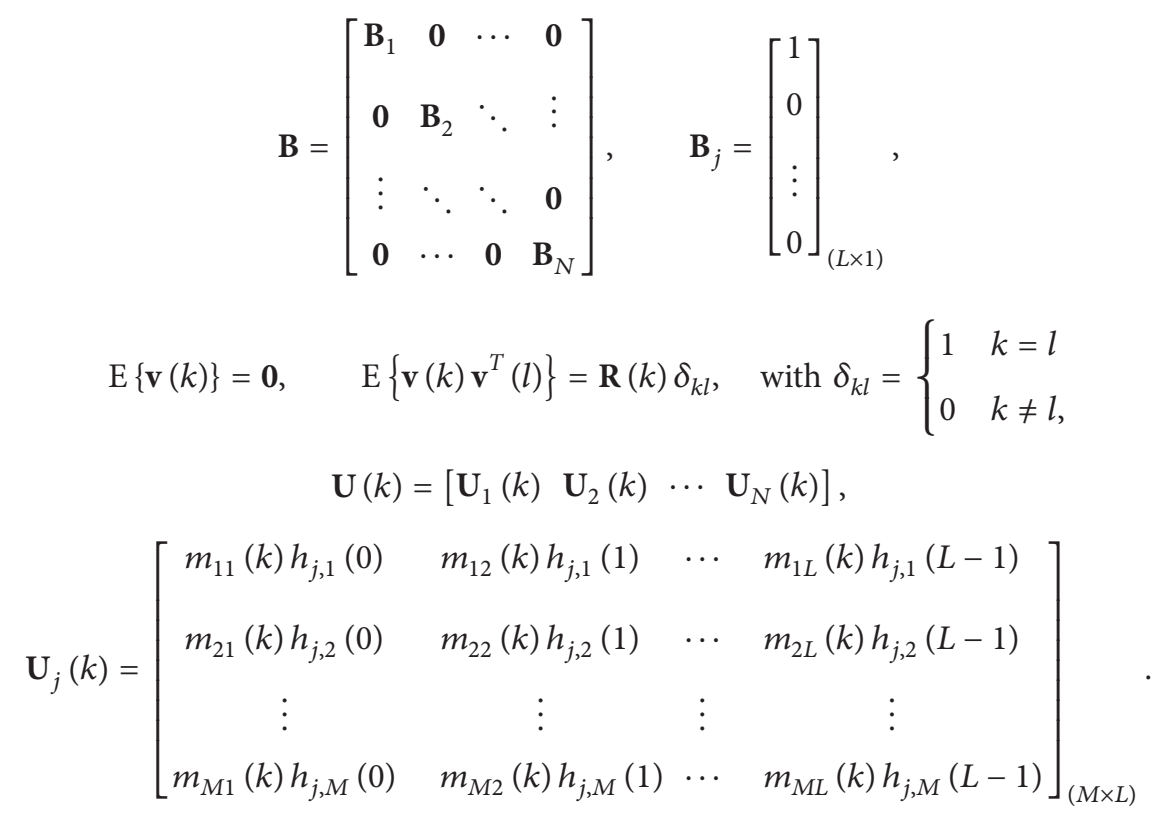

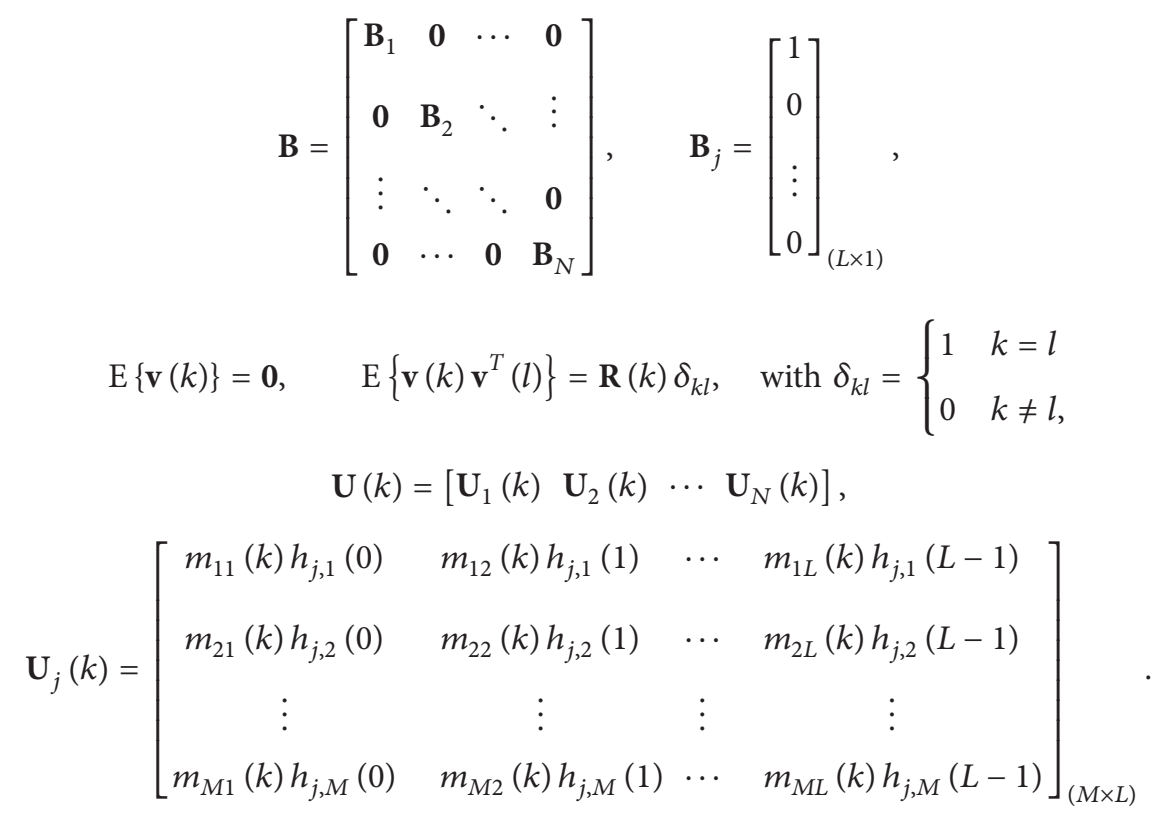

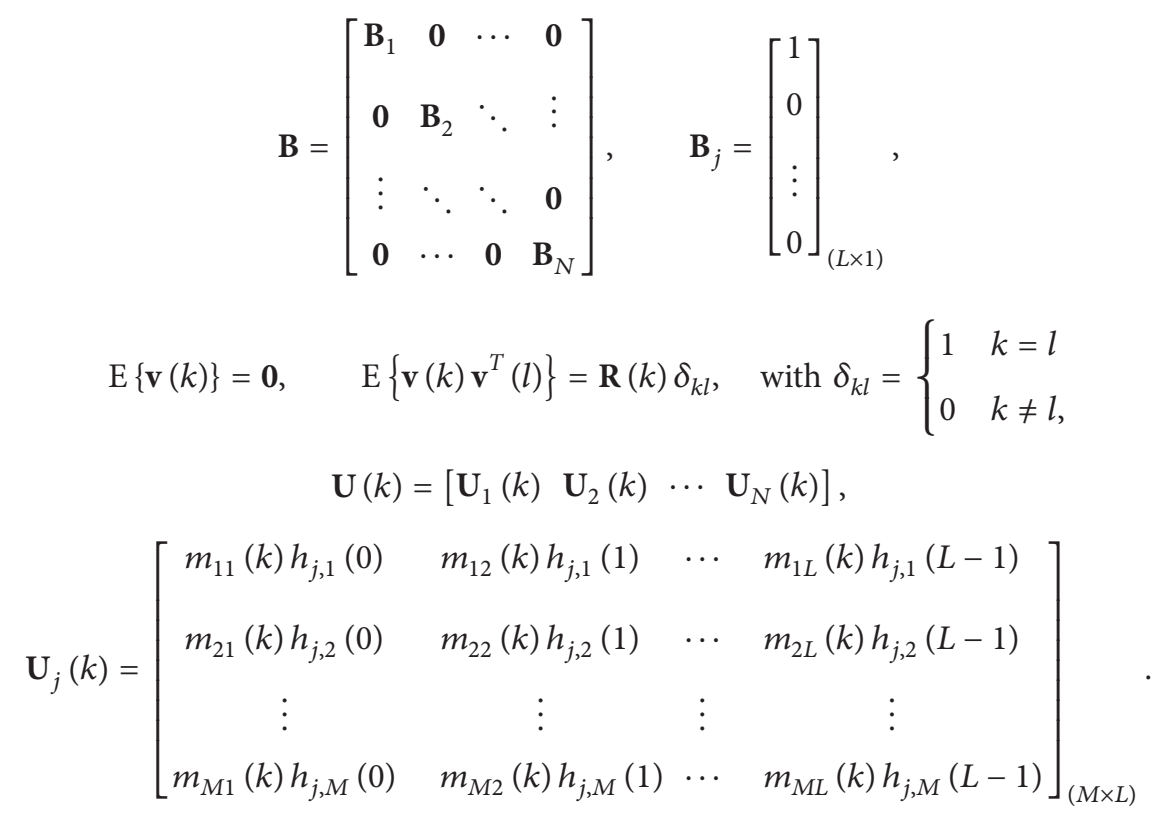

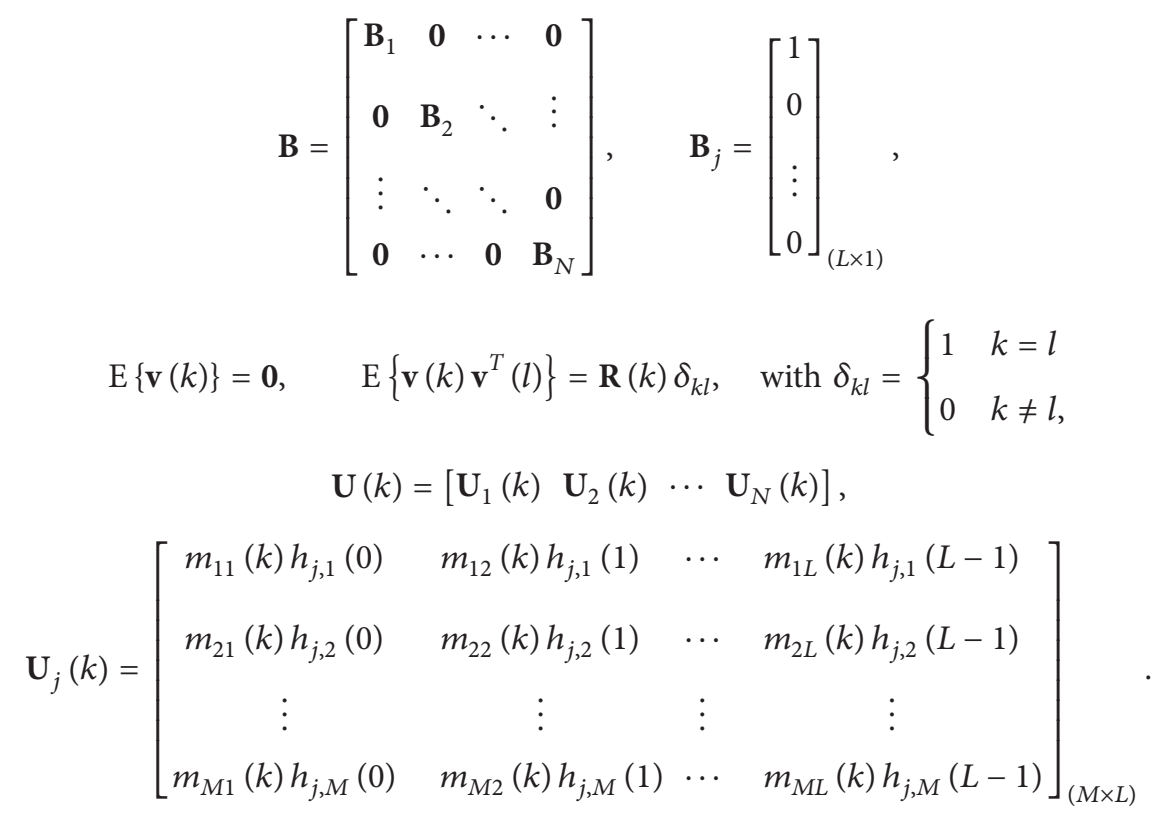

where

\section{Symbol Estimation Algorithm of MIMO UWA Communication System}

During the process of SMN modeling, we can see that $w_{j}(k-1)=s_{j}(k)=x_{(j-1) L+1}(k), j=1,2, \ldots, N$. For binary phase shift keying (BPSK) modulation system, the transmitted symbol is taken independently and equally from $\{1,-1\}$; hence, it is clear that the mean of $w_{j}(k)$ is 0 and the variance is 1 . Based on the SMN model (7), we can obtain the state estimation according to the optimal filtering algorithm. Because of the correspondence between the transmitted signals of $N$ transducer sources and the state vector $\mathbf{x}(k)$, the estimation of the transmitted signals can be acquired easily. For convenience of understanding, the algorithm is listed as follows.
The Optimal Recursive Filtering Algorithm for SMN. Consider

$$
\begin{gathered}
\mathbf{x}(k / k-1)=\mathbf{A} \mathbf{x}(k-1 / k-1), \\
\mathbf{x}(k / k)=\mathbf{x}(k / k-1) \\
\quad+\mathbf{K}(k)[\mathbf{z}(k)-\mathbf{M}(k) \mathbf{x}(k / k-1)], \\
\mathbf{P}(k / k-1)=\mathbf{A} \mathbf{P}(k-1) \mathbf{A}^{\mathrm{H}}+\mathbf{B} \mathbf{Q} \mathbf{B}^{\mathrm{H}}, \\
\mathbf{P}(k)=[\mathbf{I}-\mathbf{K}(k) \mathbf{M}(k)] \mathbf{P}(k / k-1), \\
\mathbf{K}(k)=\mathbf{P}(k / k-1) \mathbf{M}^{\mathrm{H}}(k) \mathbf{R}_{L}^{-1}(k),
\end{gathered}
$$




$$
\begin{gathered}
\mathbf{S}(k)=\mathbf{A} \mathbf{S}(k-1) \mathbf{A}^{\mathrm{H}}+\mathbf{B} \mathbf{Q} \mathbf{B}^{\mathrm{H}}, \\
\mathbf{R}_{L}(k)=\mathbf{F}(k)+\mathbf{M}(k) \mathbf{P}(k / k-1) \mathbf{M}^{\mathrm{H}}(k)+\mathbf{R}(k), \\
\mathbf{F}(k)=\mathrm{E}\left\{(\mathbf{U}(k)-\mathbf{M}(k)) \mathbf{x}(k) \mathbf{x}^{\mathrm{H}}(k)(\mathbf{U}(k)-\mathbf{M}(k))^{\mathrm{H}}\right\}, \\
f_{t l}(k)=\mathrm{E}\left\{\sum_{n=1}^{N} \sum_{j=1}^{N} \sum_{m=1}^{L} \sum_{i=1}^{L} \Delta_{t i}(k) \Delta_{l m}(k)\right.
\end{gathered}
$$$$
\left.\begin{array}{l}
\cdot a_{(j-1) L+i,(n-1) L+m}(k) \\
\cdot h_{j, t}(i-1) h_{n, l}(m-1)
\end{array}\right\},
$$

where $(\cdot)^{\mathrm{H}}$ denotes Hermitian transpose of a vector or matrix, $f_{t l}(k)$ is the $(t, l)$ th element of matrix $\mathbf{F}(k)$, and $\mathbf{x}(k) \mathbf{x}^{\mathrm{H}}(k)=$ $\left\{a_{i j}(k)\right\}_{N L \times N L}$;

$$
\begin{aligned}
& \mathbf{M}(k)=\left[\begin{array}{llll}
\mathbf{M}_{1}(k) & \mathbf{M}_{2}(k) & \cdots & \mathbf{M}_{N}(k)
\end{array}\right] \text {, with } \\
& \mathbf{M}_{j}(k)=\left[\begin{array}{cccc}
\bar{m}_{11}(k) h_{j, 1}(0) & \bar{m}_{12}(k) h_{j, 1}(1) & \cdots & \bar{m}_{1 L}(k) h_{j, 1}(L-1) \\
\bar{m}_{21}(k) h_{j, 2}(0) & \bar{m}_{22}(k) h_{j, 2}(1) & \cdots & \bar{m}_{2 L}(k) h_{j, 2}(L-1) \\
\vdots & \vdots & \vdots & \vdots \\
\bar{m}_{M 1}(k) h_{j, M}(0) & \bar{m}_{M 2}(k) h_{j, M}(1) & \cdots & \bar{m}_{M L}(k) h_{j, M}(L-1)
\end{array}\right]_{(M \times L)} ; \\
& \mathrm{E}\left\{m_{i j}(k)\right\}=\bar{m}_{i j}(k) ; \quad \Delta_{i j}(k)=m_{i j}(k)-\bar{m}_{i j}(k) .
\end{aligned}
$$

\section{Performance Analysis}

4.1. Optimality. The recursive filtering algorithm proposed in this paper is based on the well-known projection theorem [23], which is optimal in the sense of linear minimum variance. Therefore, the theoretical performance of the estimation algorithm is guaranteed such as the optimality and the convergence rate, which will be illustrated by the simulation examples in the next section. Furthermore, the model and the corresponding algorithm can be more flexibly extended to solve some practical systems under complex environment interfered by multiplicative noises, such as the symbol estimation problem of the MIMO UWA communication system with packet dropout and the estimation of Doppler frequency offset in MIMO OFDM systems.

4.2. Recursive Computation. The algorithm is computed recursively with computational complexity $O\left(N^{2}\right)$, where $N$ is the number of the transducers, that is, the dimension of the state vector. Since the algorithm has low computation cost, it can track the fast time-varying UWA channel dynamically, which allows the UWA channel gain changing even over one symbol interval, while the equalization algorithm cannot. The convergence rate of the proposed algorithm will be verified by the simulation in the next section. The above advantages of the new algorithm will better adapt to the demand of the future high-speed underwater acoustic communication system.

\section{Simulation and Comparison}

It is known that UWA channels change randomly over time, so we have some simulation results for the optimal recursive filtering algorithm when the multiplicative noise
$\mathbf{U}(k)$ is a general stochastic matrix with every component related to each other at the same time index. In addition, based on the characteristics of UWA channel, we assume that it obeys Rayleigh distribution. Since the multiplicative noise can describe the fading, nonlinearity, and distortion of the channel, it is reasonable to model the Rayleigh fading channel. The received signal obtained from the multipath Rayleigh fading channel is estimated by the proposed optimal filtering algorithm in this paper and the adaptive equalization algorithm from [12], respectively, and the BER performance of these two algorithms is compared under the same signal to noise ratio (SNR) in Figures 4, 5, and 6.

5.1. Simulation of the Proposed Optimal Filtering Algorithm. For $2 \times 2$ UWA communication system with 2 transmitters and 2 receivers, the least square (LS) estimation of the channel impulse response can be obtained by the method in [24]. Set $L=5$. Based on the characteristics of UWA channel, we presume that the mathematic expectation of the multiplicative noise $m_{i j}(k)$ is 0.9 ; the variance and the covariance of each component in $\mathbf{U}_{j}(k)$ are 0.02 .

Figure 1 depicts the plot of BER versus SNR by BPSK modulation using the proposed algorithm to estimate transmitted signals from the two transducer sources. Figure 2 shows BER versus SNR by quadrature phase shift keying (QPSK) modulation from the two transducer sources using the present algorithm.

From Figures 1 and 2, we can see that BER is about $10^{-3}$ when SNR is $16 \mathrm{~dB}$; therefore, the simulation results show that the proposed optimal filtering algorithm of SMN can effectively estimate the transmitted characters, and it is suitable for solving the symbol detection problem of MIMO UWA communication system. 


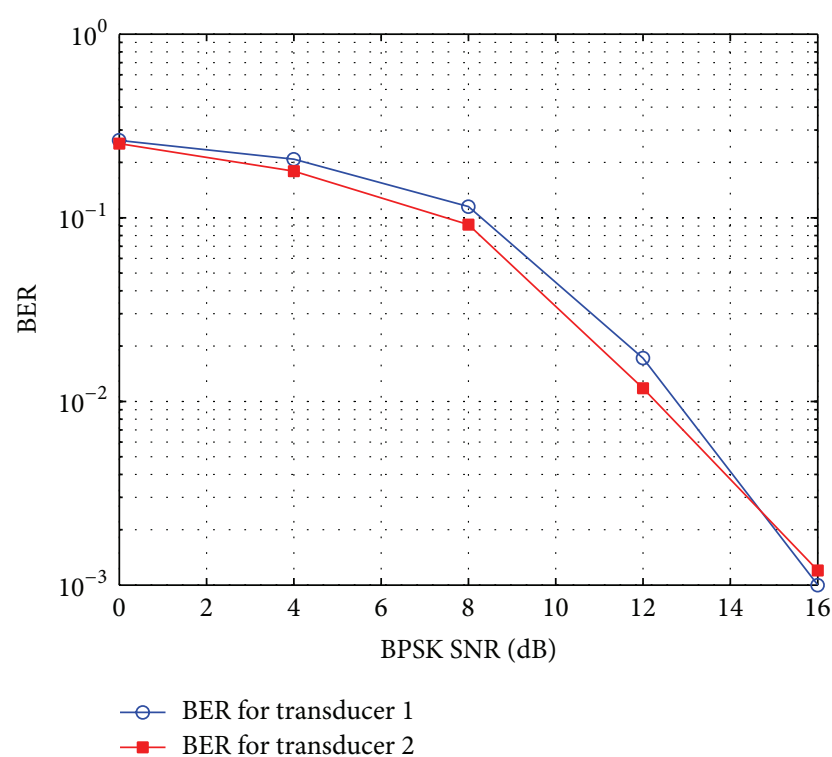

FIGURE 1: BER versus SNR of two transducer sources for BPSK modulation.

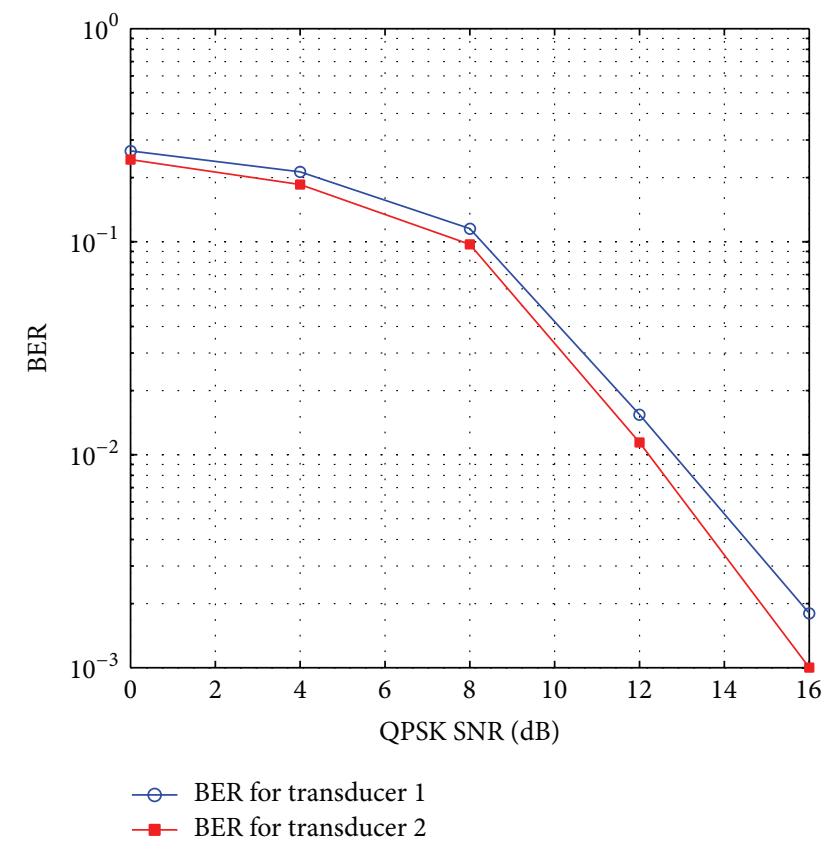

FIGURE 2: BER versus SNR of two transducer sources for QPSK modulation.

5.2. Comparison with Other Related Algorithms. The literature [25] describes the time-varying characteristics of Rayleigh fading channel from the perspective of UWA communication and utilizes a simplified Rayleigh statistical model to simulate the random time-varying and fading UWA channel, which is a generally accepted UWA channel model compared with the actual underwater channel.

In this paper, UWA channel is taken as a Rayleigh fading channel with three paths; transmitted signals are modulated

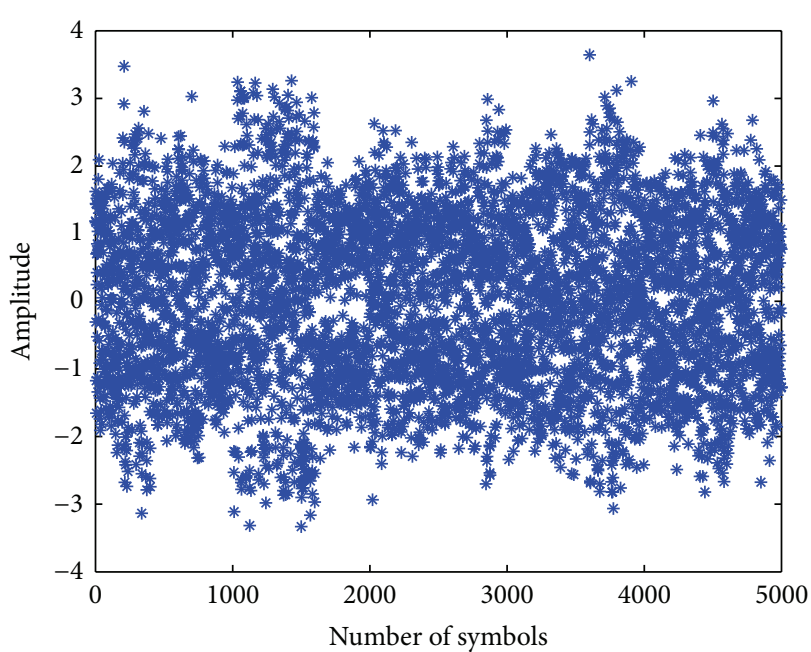

FIGURE 3: Scatter plot of received baseband signals from Rayleigh fading channel with three paths.

by BPSK; the carrier frequency is $5 \mathrm{kHz}$; the sampling frequency is $20 \mathrm{kHz}$; the symbol rate is $1 \mathrm{k}$ baud; the multipath delay vector is $[0,1,2] \mathrm{ms}$; and the multipath power vector is $[0,-1,-9] \mathrm{dB}$. Set the channel parameters change every 200 symbol intervals. The received signals obtained from this multipath Rayleigh fading channel are processed by the adaptive equalization algorithm from [12] and the proposed optimal filtering algorithm in this paper, respectively. The relevant parameters of the adaptive equalization algorithm can be obtained from [12]. Since the equalization algorithm [12] is suitable for the single input single output (SISO) UWA communication system, set $N=1$ and $M=1$ in the proposed optimal filtering algorithm. The following simulation results compare the performance of the optimal filtering algorithm and the equalization algorithm in terms of the optimality, convergence rate, and so forth.

Figure 3 is the scatter plot of received baseband signals obtained from the Rayleigh fading channel model with three paths.

Figures 4 and 5 are the scatter plots of signals processed by the adaptive equalization algorithm [12] and the proposed optimal filtering algorithm, respectively.

Figure 6 depicts BER versus SNR of the two algorithms for comparison of the estimation performance.

From Figures 4 and 5, we can see that the severely aliasing received signals can be clearly separated by the adaptive equalization algorithm [12] and the proposed optimal filtering algorithm, but the convergence rate of the proposed algorithm is faster than that of the equalization algorithm which converges after about 500 symbols as shown in Figure 4. From Figure 6, we can see that BER of the proposed algorithm is lower than that of the equalization algorithm [12], which confirms the optimality of the proposed algorithm. Therefore, the effectiveness of the proposed optimal filtering algorithm for the UWA communication system is validated. 


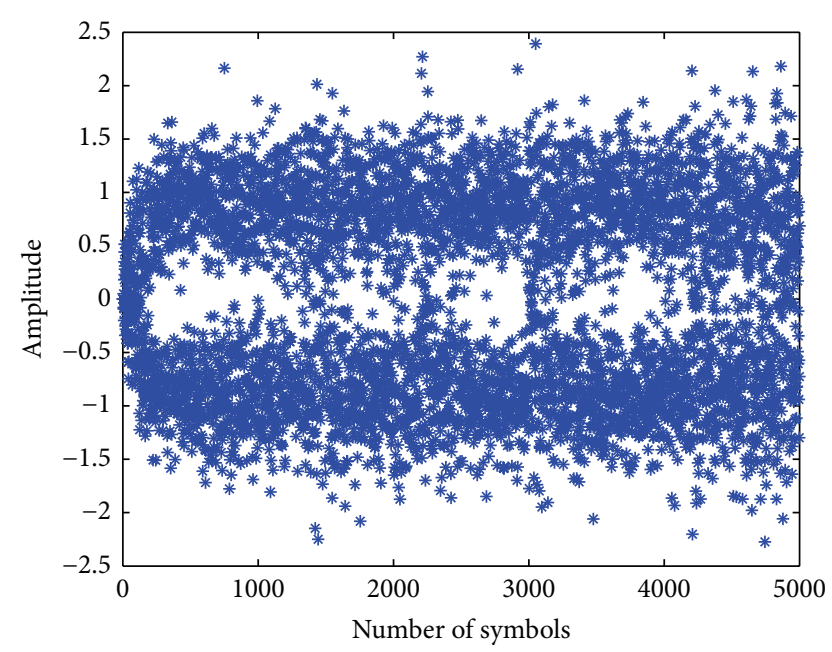

FIGURE 4: Scatter plot of signals processed by the adaptive equalization algorithm.

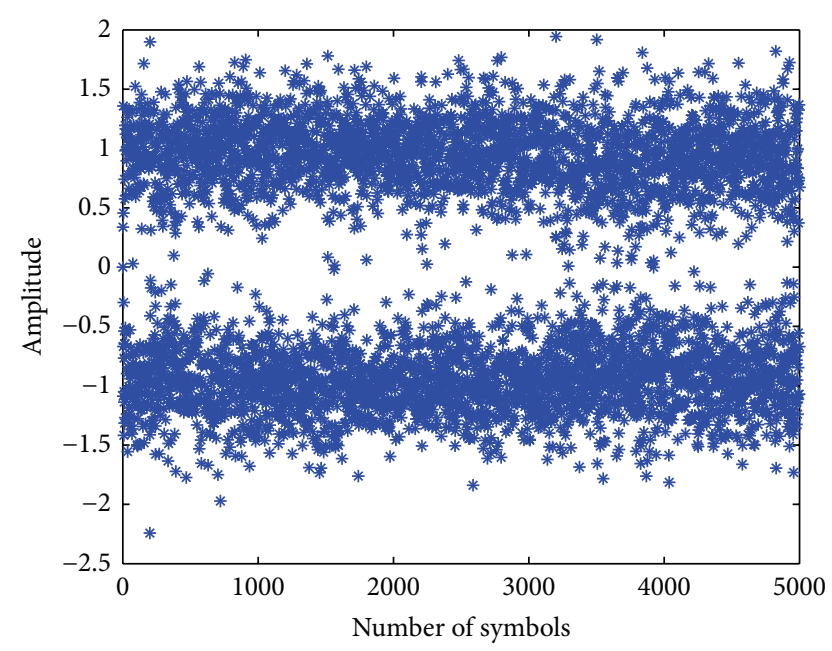

FIGURE 5: Scatter plot of signals processed by the proposed optimal filtering algorithm.

\section{Conclusions}

In this paper, the complicated and time-varying MIMO underwater acoustic channel is characterized by an SMN model with a random general multiplicative noise matrix. Based on the system model, the estimation of UWA symbols is derived through the optimal recursive filtering algorithm of SMN. The algorithm is optimal in the sense of linear minimum variance with recursive calculation process, which provides faster convergence rate and lower computational complexity compared with the typically used equalization algorithm in underwater acoustic communication. The proposed recursive optimal filtering algorithm can track the random time-varying UWA channels dynamically; thereby the SMN channel model in association with the optimal filtering algorithm could meet the requirements of high speed UWA communication system in complex environment. Simulation results show that the proposed method can effectively

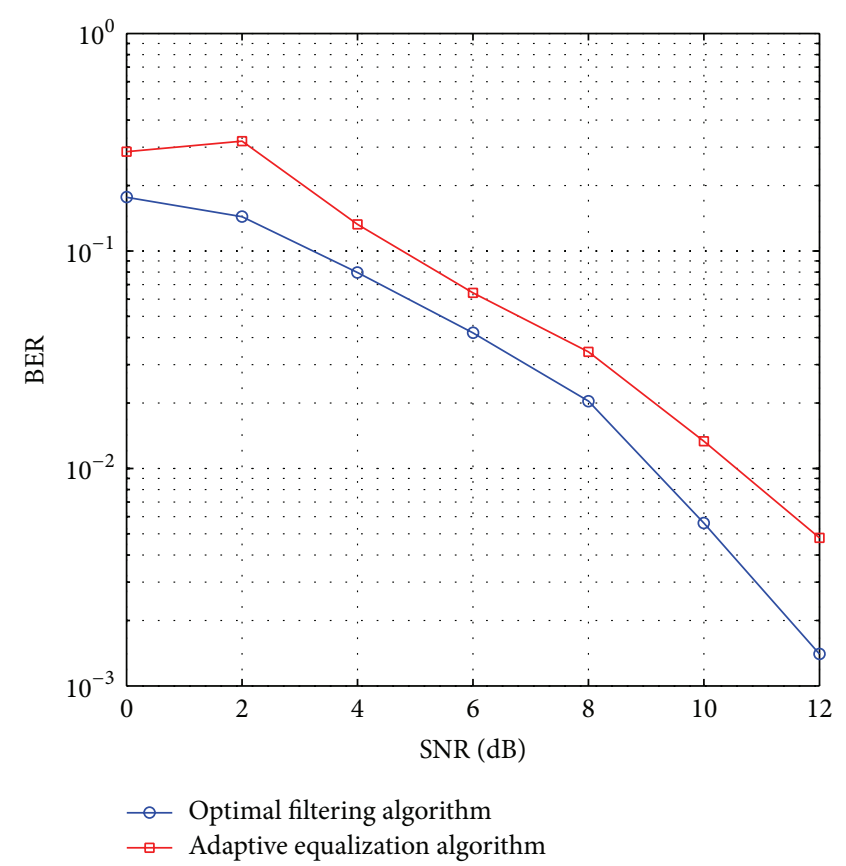

FIGURE 6: BER versus SNR of the equalization algorithm [12] and the optimal filtering algorithm.

estimate the transmitted symbols corrupted by the stochastic multiplicative noise in complex communication environment with random time-varying UWA channels. Furthermore, the algorithm can be flexibly extended for solving the joint channel and symbol estimation problem of MIMO UWA communication system contaminated by the multiplicative interference. Overall the SMN model has higher practical merits and wider range of applications.

\section{Conflict of Interests}

The authors declare that there is no conflict of interests regarding the publication of this paper.

\section{Acknowledgments}

This project is supported by the National Natural Science Foundation of China (Grants nos. 61132005, 51279185, and 61401111) and Shandong Natural Science Foundation of China (Grant no. ZR2010DQ003).

\section{References}

[1] A. Stefanov and M. Stojanovic, "Design and performance analysis of underwater acoustic networks," IEEE Journal on Selected Areas in Communications, vol. 29, no. 10, pp. 2012-2021, 2011.

[2] M. Chitre, S. Shahabudeen, and M. Stojanovic, "Underwater acoustic communications and networking: recent advances and future challenges," Marine Technology Society Journal, vol. 42, no. 1, pp. 103-116, 2008. 
[3] C. Qi, X. Wang, and L. Wu, "Underwater acoustic channel estimation based on sparse recovery algorithms," IET Signal Processing, vol. 5, no. 8, pp. 739-747, 2011.

[4] A. K. Morozov, J. C. Preisig, and J. C. Papp, "Investigation of mode filtering as a preprocessing method for shallow-water acoustic communications," IEEE Journal of Oceanic Engineering, vol. 35, no. 4, pp. 744-755, 2010.

[5] J. Tao, Y. R. Zheng, C. S. Xiao et al., "Time-domain receiver design for MIMO underwater acoustic communications," in Proceedings of the OCEANS 2008, pp. 1-6, September 2008.

[6] J. Tao, Y. R. Zheng, C. S. Xiao, and T. C. Yang, "Robust MIMO underwater acoustic communications using turbo block decision-feedback equalization," IEEE Journal of Oceanic Engineering, vol. 35, no. 4, pp. 948-960, 2010.

[7] J. Zhang and Y. R. Zheng, "Frequency-domain turbo equalization with soft successive interference cancellation for single carrier mimo underwater acoustic communications," IEEE Transactions on Wireless Communications, vol. 10, no. 9, pp. 2872-2882, 2011.

[8] R. Arablouei and K. Doançay, "Low-complexity adaptive decision-feedback equalization of MIMO channels," Signal Processing, vol. 92, no. 6, pp. 1515-1524, 2012.

[9] M. Stojanovic, "Adaptive channel estimation for underwater acoustic MIMO OFDM systems," in Proceedings of the IEEE 13th Digital Signal Processing Workshop and 5th IEEE Signal Processing Education Workshop, pp. 132-137, IEEE, Marco Island, Fla, USA, January 2009.

[10] J. Huang, S. Zhou, J. Huang, C. R. Berger, and P. Willett, "Progressive inter-carrier interference equalization for OFDM transmission over time-varying underwater acoustic channels," IEEE Journal on Selected Topics in Signal Processing, vol. 5, no. 8, pp. 1524-1536, 2011.

[11] K. Pelekanakis and A. B. Baggeroer, "Exploiting space-timefrequency diversity with MIMO-OFDM for underwater acoustic communications," IEEE Journal of Oceanic Engineering, vol. 36, no. 4, pp. 502-513, 2011.

[12] H.-J. Li and C. Sun, "Low computational complexity adaptive equalization algorithm for time-variety underwater acoustic channels," Journal of System Simulation, vol. 19, no. 23, pp. 54695472, 2007.

[13] G. Zhang, J. M. Hovem, H. Dong, S. Zhou, and S. Du, "An efficient spread spectrum pulse position modulation scheme for point-to-point underwater acoustic communication," Applied Acoustics, vol. 71, no. 1, pp. 11-16, 2010.

[14] Y. R. Zheng, C. Xiao, T. C. Yang, and W.-B. Yang, "Frequencydomain channel estimation and equalization for shallow-water acoustic communications," Physical Communication, vol. 3, no. 1, pp. 48-63, 2010.

[15] L. Zhao and J. Ge, "Iterative receiver in time-frequency domain for underwater acoustic communications," Procedia Engineering, vol. 30, pp. 844-851, 2012.

[16] S. Loyka and C. D. Charalambous, "On the compound capacity of a class of MIMO channels subject to normed uncertainty," IEEE Transactions on Information Theory, vol. 58, no. 4, pp. 2048-2063, 2012.

[17] S. Karabchevsky, D. Kahana, O. Ben-Harush, and H. Guterman, "FPGA-based adaptive speckle suppression filter for underwater imaging sonar," IEEE Journal of Oceanic Engineering, vol. 36, no. 4, pp. 646-657, 2011.

[18] N. Brahim, D. Guériot, S. Daniel, and B. Solaiman, “3D reconstruction of underwater scenes using image sequences from acoustic camera," in Proceedings of the IEEE OCEANS 2010, pp. 1-8, Sydney, Australia, May 2010.

[19] Y.-S. Hsu, K. T. Wong, and L. Yeh, "Mismatch of near-field bearing-range spatial geometry in source-localization by a uniform linear array," IEEE Transactions on Antennas and Propagation, vol. 59, no. 10, pp. 3658-3667, 2011.

[20] T. Chen, "The past present, and future of image and multidimensional signal processing," IEEE Signal Processing Magazine, vol. 15, no. 2, pp. 21-58, 1998.

[21] D. S. Chu, Z. F. Yin, and L. Zhang, "Study of symbol estimation algorithm for underwater acoustic communication with multiplicative noise model," Periodical of Ocean University of China, vol. 42, no. 1, pp. 149-152, 2012.

[22] S. H. Huang, J. Tsao, T. C. Yang, and S. W. Cheng, "Model-based signal subspace channel tracking for correlated underwater acoustic communication channels," IEEE Journal of Oceanic Engineering, vol. 39, no. 2, pp. 343-356, 2014.

[23] L. Zhang and X.-D. Zhang, "An optimal filtering algorithm for systems with multiplicative/additive noises," IEEE Signal Processing Letters, vol. 14, no. 7, pp. 469-472, 2007.

[24] J. Tao, Y. R. Zheng, C. S. Xiao, T. C. Yang, and W.-B. Yang, "Channel estimation, equalization and phase correction for single carrier underwater acoustic communications," in Proceedings of the MTS/IEEE Kobe Techno-Ocean (OCEANS '08), pp. 1-6, IEEE, Kobe, Japan, April 2008.

[25] M. Pajovic and J. C. Preisig, "Performance analysis of least squares-based multichannel decision feedback equalization of time-varying channels," in IEEE Global Communications Conference (GLOBECOM '13), pp. 3312-3317, Atlanta, Ga, USA, December 2013. 


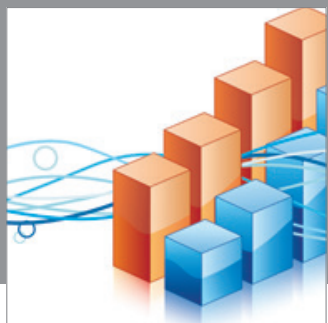

Advances in

Operations Research

mansans

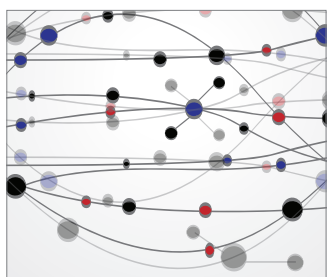

The Scientific World Journal
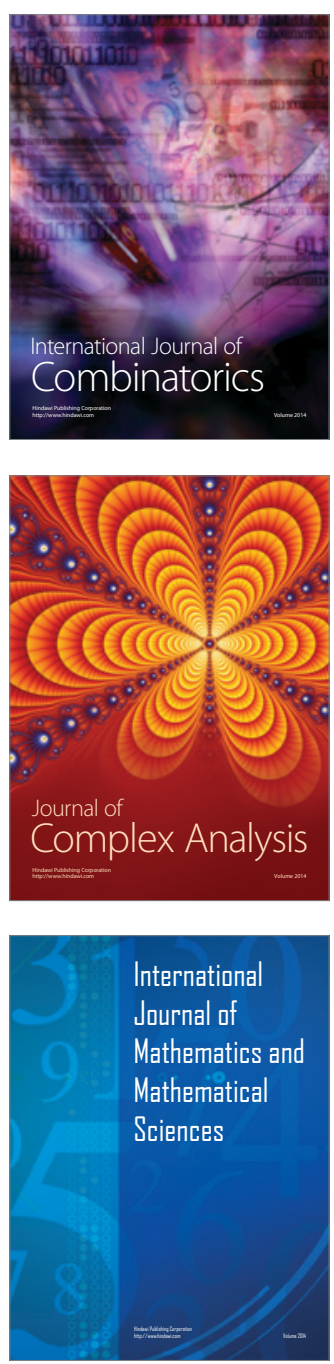
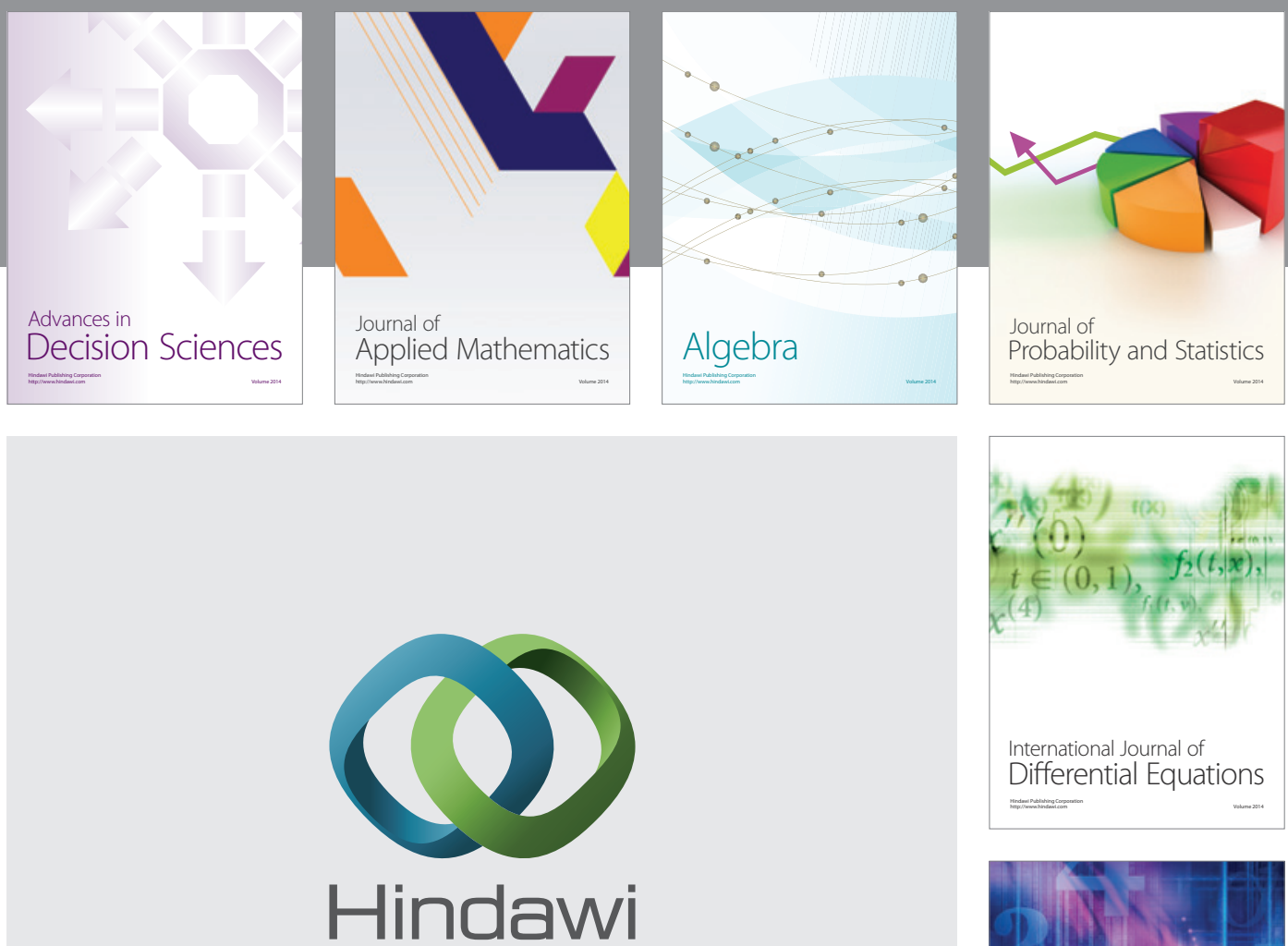

Submit your manuscripts at http://www.hindawi.com
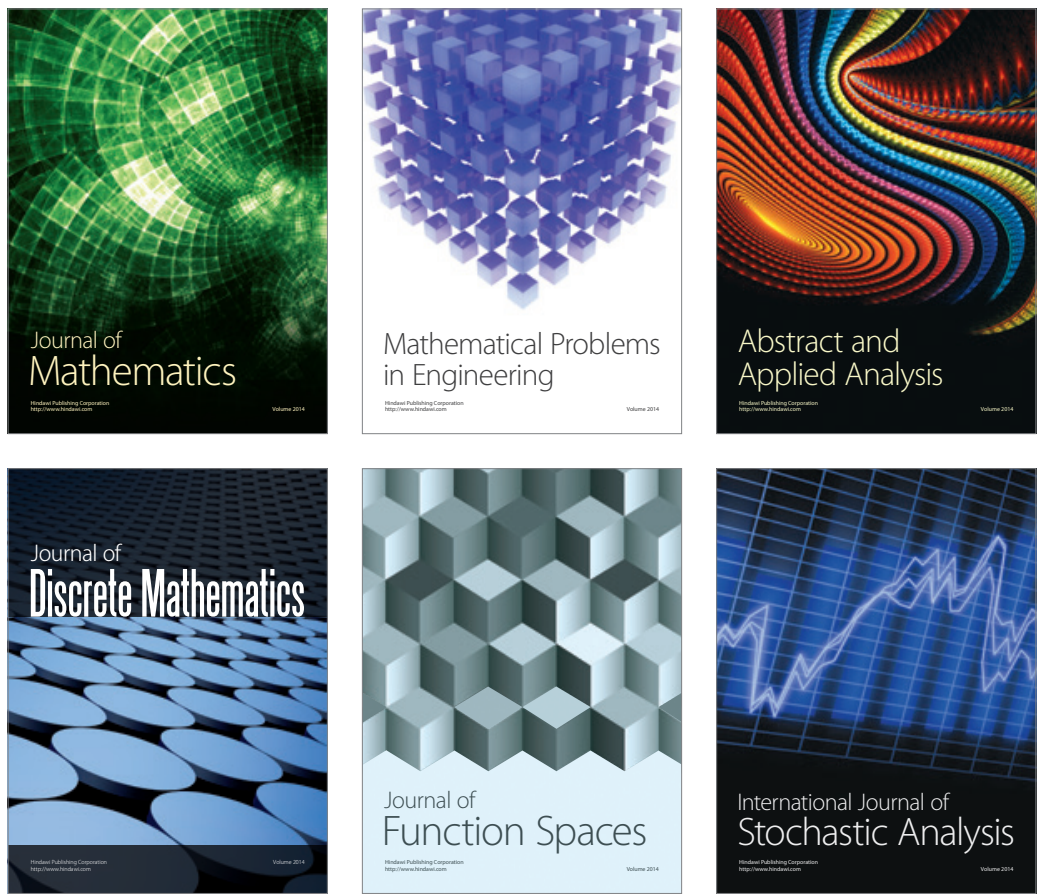

Journal of

Function Spaces

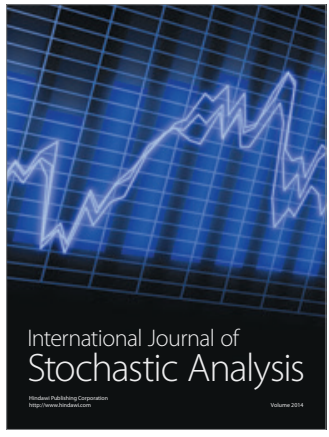

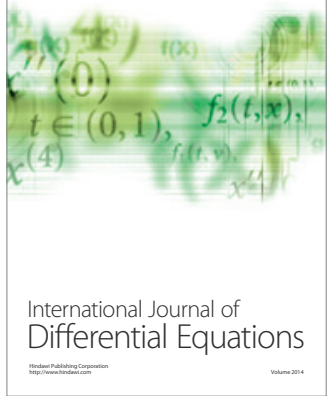
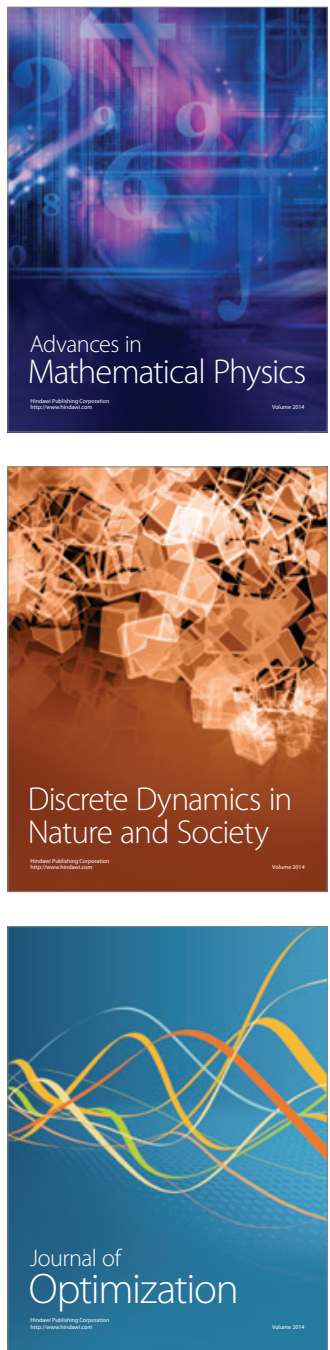